\title{
Concurrency Control in Real-Time Object-Oriented Systems: The Affected Set Priority Ceiling Protocols*
}

\author{
Michael Squadrito, Levon Esibov, Lisa C. DiPippo, Victor F. Wolfe, and Gregory Cooper (lastname@cs.uri.edu) \\ University of Rhode Island, USA \\ Bhavani Thurasignham thura@mitre.org, Peter Krupp pck@mitre.org MITRE Corporation, Bedford, MA USA \\ Michael Milligan milliganm@hanscom.af.mil Hanscom AFB USA \\ Russell Johnston russ@nosc.mil U.S. Navy NRaD, San Diego, CA USA
}

\begin{abstract}
This paper presents the Affected Set Priority Ceiling Protocols (ASPCP) for concurrency control in real-time object-oriented systems. These protocols are based on a combination of semantic locking and priority ceiling techniques. This paper shows that the ASPCP protocols provide higher potential concurrency for object-oriented systems than existing Priority Ceiling protocols, while still bounding priority inversion and preventing deadlock.
\end{abstract}

\section{Introduction}

The advent of real-time object-oriented (RTOO) systems, such as Real-Time CORBA middleware [6, 3] and RTOO databases [2], poses the need to control concurrent access to objects under real-time requirements. In a real-time database, the concurrency control technique manages concurrent access by transactions to data objects. In a CORBA system, the middleware must control concurrent access by remote clients to CORBA objects.

Concurrency control techniques for RTOO systems must satisfy more requirements than traditional nonreal-time concurrency control techniques because they must also meet timing constraints. Among the most important traditional non-real-time requirements are that the technique provides: high concurrency to maximize average throughput; deadlock treatment that either prevents, avoids, or breaks deadlocks; and logical consistency, such as mutual exclusion or serializability, so that all constraints on the values of object attributes are met. In real-time concurrency control, there are similar requirements, along with the requirement that the technique should support predictable execution, such as bounded blocking times for locks. Providing predictable blocking times involves, among

${ }^{*}$ This work has been supported by the U.S. Office of Naval Research grant N000149610401. other things, bounding priority inversion that occurs when a lower priority task blocks a higher priority task [4].

In this paper we describe two new techniques for concurrency control in RTOO systems: one for singlenode RTOO systems, such as real-time databases; and one for distributed RTOO systems, such as RT CORBA middleware. Both techniques are based on the priority ceiling (PCP) family of protocols [4]. Our single-node RTOO concurrency control technique is called the Affected Set Priority Ceiling Protocol (ASPCP), and the multi-node technique is called the Distributed ASPCP (DASPCP).

Both protocols use PCP techniques while exploiting the semantics of the object-oriented paradigm. They do this through method-level locking, where a transaction or client locks a particular method on an object. Method locking is a finer granularity of object lock than exclusive locking, where the transaction/client locks the entire object exclusively; or read/write locking, in which the transaction/client locks the ability to read or write the entire object. Our protocols are a form of semantic real-time object-based concurrency control [2] that uses the semantics of the object to determine the compatibility of method locks. In particular, these two protocols use the semantics of the affected sets of methods [1] to determine the compatibility of method locks. The result is a pair of protocols that, like the other PCPs, prevent deadlock and bound priority inversion [4], and do so while allowing more potential concurrency in RTOO systems than other PCPs.

Section 2 presents the RTOO model that we assume. It also summarizes the original work on singlenode and distributed PCP techniques to establish the framework for our techniques. Section 3 presents our ASPCP protocol for single-node RTOO systems, such as real-time object-oriented databases. Sec- 
tion 3 also shows how the ASPCP can lower priority ceilings for objects and therefore increase potential concurrency compared to the original PCP techniques. Furthermore, the section shows that ASPCP still prevents deadlock and tightly bounds priority inversion. Section 4 presents the DASPCP for distributed RTOO systems, such as Real-Time CORBA middleware. Like Section 3 , this section demonstrates DASPCP's increased potential concurrency while still maintaining deadlock freedom and priority inversion bounds. Section 5 summarizes.

\section{Background}

Previous work in object-based semantic real-time concurrency control [2] and in priority ceiling protocols [4] has led to our development of the Affected Set Priority Ceiling Protocols. Our previous work in semantic concurrency control [2] indicates that using object semantics to increase concurrency in a real-time database can enhance real-time performance. However, in general, the semantic concurrency control techniques can be complex and do not necessarily bound priority inversion nor prevent deadlock. Fortunately, priority ceiling protocols have been proven to bound priority inversion and prevent deadlock [4] in certain systems. By combining object semantics with priority ceiling techniques, we developed the ASPCP protocols presented in this paper.

This section first describes the model of RTOO systems that we assume and also indicates how the model supports semantic real-time concurrency control for object-oriented systems. We then summarizes previous work by Rajkumar, Sha, et. al, in developing the priority ceiling protocol and the distributed priority ceiling protocol - both of which provide the framework for our object-based ASPCP protocols.

\subsection{Real-Time Object-Oriented System Model}

A RTOO system consists of objects, some of which manage shared resources. The model of a real-time object that we use in this paper is derived from the RTSORAC model [2] for real-time object-oriented databases.

Our RTOO system object model extends the traditional object-oriented notion of an object to include attributes that have a value, a timestamp and an amount of accumulated imprecision. The imprecision that is recorded accumulates due to the potential relaxation of serializability by semantic concurrency control [2]. Objects also include constraints and a compatibility function. The constraints can be placed on the attributes to express logical and temporal correctness of the object.
The user-defined compatibility function determines how the methods of the object may interleave. It is through this function that the object designer expresses the semantics of allowable concurrency. The flexibility of the compatibility function allows the object designer to specify different levels of concurrency for different objects. For instance, one object may require serializability, while another object may tolerate a less restrictive form of correctness. To enforce serializability the object designer may use affected set semantics [1] to determine compatibility. A method's Read Affected Set $(R A)$ is the set of the object's attributes that the method reads. A method's Write Affected Set (WA) is the set of the object's attributes that the method writes. Under affected set semantics, two methods $m_{1}$ and $m_{2}$ are compatible if and only if:

$$
\begin{gathered}
\left(W A\left(m_{1}\right) \cap W A\left(m_{2}\right)=\emptyset\right) \wedge\left(W A\left(m_{1}\right) \cap R A\left(m_{2}\right)=\emptyset\right) \wedge \\
\left(R A\left(m_{1}\right) \cap W A\left(m_{2}\right)=\emptyset\right)
\end{gathered}
$$

Note that defining lock compatibility based on these affected set semantics has been proven to produce serializable object schedules [1].

A less restrictive form of correctness may be needed to express the trade-off between temporal and logical consistency. In such cases, the semantics of compatibility between methods are based on dynamic information, including current temporal consistency and imprecision of data. For example, if a method $m_{1}$ that reads an attribute $a$ is currently executing, it would violate the logical consistency of $m_{1}$ 's return value if another method $m_{2}$ that writes $a$ were to execute. However, if the timing constraint on $a$ has been violated, i.e. it has become old, then allowing $m_{2}$ to execute would restore the temporal consistency of $a$. When determining each potential allowable interleaving of method executions, the compatibility function can also examine the amount of imprecision that could be introduced by the possible interleaving.

We developed a semantic locking concurrency control technique [2] that utilizes the full semantics of the compatibility function to express the trade-off between temporal and logical consistency. It has been shown to bound the imprecision that is accumulated due to nonserializable method interleavings [2]. While this semantic locking concurrency control technique provides the potential for increased concurrency for meeting more transaction deadlines, it suffers from unbounded priority inversion and the possibility of deadlock, both of which can affect the system's predictability and its ability to meet timing constraints.

\subsection{Priority Ceiling Protocols}

A priority ceiling protocol [4] uses information about the way in which transactions intend to use the 
resources of the system to bound priority inversion and to prevent deadlock. It is based on the assumption about the system that every object and every transaction in the system is known a priori. Thus, no dynamic information may be used to determine the semantics of concurrency control.

There are three basic steps to any of the priority ceiling protocols:

1. Before running, the protocol defines a priority ceiling for each critical section that may be locked. The granularity of these critical sections is the core difference among the various priority ceiling protocols.

2. At run-time, when a transaction $T$ requests a lock, the lock can be granted only if $T$ 's priority is strictly higher than the ceiling of locks held by all other transactions.

3. If transaction T's lock request is denied because $T_{\text {low }}$ (a lower priority transaction) holds a lock with priority ceiling equal to or greater than $T$ 's priority, $T_{\text {low }}$ inherits the priority of $T$ until $T_{\text {low }}$ 's lock is released.

Note that no checking of conflict is necessary when granting a lock. This is because conflict in a priority ceiling protocol is captured in the definition of the priority ceiling.

Each of the protocols from Rajkumar, Sha et al. that we describe below follow these basic steps. The difference among them arises in how conflict is defined among locks and thus, how priority ceiling is defined. We will describe how priority ceiling is defined in each protocol.

The Basic Priority Ceiling Protocol. In the basic priority ceiling protocol [4], exclusive locks are placed on entire objects. Thus, the critical section requires a lock on the entire object. The priority ceiling of a lock is defined as the priority of the highest priority transaction that will ever use this lock. A transaction $T$ can lock a critical section only if it passes the test of Step 2 (above): The priority of transaction $T$ must be strictly higher than the priority ceiling of locks held by all other transactions.

The Read/Write Priority Ceiling Protocol. In a database that allows select, insert, and update functionality, a division can be made between read and write operations. Instead of acquiring an exclusive lock on an entire object, a transaction can request read and write locks. Bounding priority inversion and preventing deadlock with read/write locking has been addressed by the read/write priority ceiling protocol [4].

In the Read/Write priority ceiling protocol, since each object can allow both readers and writers, each object requires two static priority ceilings, and the system dynamically determines which of these two priority ceilings to use as the overall read/write priority ceiling for the object as follows:

1. The write priority ceiling is set equal to the highest priority transaction that will ever write the object.

2. The absolute priority ceiling is set equal to the highest priority transaction that will ever read or write the object.

3. The read/write priority ceiling is set at run-time. If a transaction is allowed to read an object, the $\mathrm{read} /$ write priority ceiling is set equal to the write priority ceiling. If a transaction is allowed to write an object, the read/write priority ceiling is set equal to the absolute priority ceiling.

In the read/write priority ceiling protocol, a critical section is a read/write lock. A transaction $T$ can lock a critical section only if it passes the following test:

The priority of transaction $T$ must be strictly higher than the read/write priority ceiling of locks held by all other transactions.

\subsection{Distributed Priority Ceiling Protocol.}

The Distributed Priority Ceiling Protocol (DPCP) [4] allows tasks to lock objects on remote nodes.

DPCP Terminolgy and Assumptions. An object lock that is accessed by tasks from remote processors is referred to as a global lock. If the lock is accessed only by tasks on its node, it is referred to as a local lock. A critical section guarded by a global lock is referred to as a global critical section (GCS). A critical section guarded by a local lock is referred to as a local critical section (LCS). A task $T$ executes its non-critical-section code and LCS's on its host processor. A task's GCS's may be bound and executed on a processor(s) different than the task's host processor. All GCS's that are controlled by the same lock must be bound to the same processor. DPCP prohibits a mixed nesting of LCSs and GCSs, and GCSs at different nodes within a task. 
DPCP Priority Ceiling. The base priority ceiling, $P G$, is a fixed priority, greater than or equal to the priority assigned to the highest priority task in the system (in the examples of this paper we will make $P G$ equal to the highest priority of a task in the system). The priority ceiling of a local lock is the highest priority of all tasks that access it. The priority ceiling of a global lock is the highest priority of all tasks that access it plus $P G$.

DPCP Priority Assignment. A GCS that is generated by task $T$, is assigned a priority equal to the sum of the base priority ceiling $P G$ and the priority of $T$.

Priority Ceiling Protocol. Each processor runs the priority ceiling protocol on the LCSs and GCS's by considering each thread of execution for executing a GCS as a "task".

DPCP Example. The DPCP is a complicated protocol with many cases to consider. The following example shows some of the cases. For a more detailed example of the application of DPCP, we refer reader to Rajkumar's work [4].

Consider a distributed system with two nodes. The application consists of three tasks and two objects ( $O_{\text {track } 1}$ and $\left.O_{\text {track } 2}\right)$, guarded by 2 locks $\left(L_{1}\right.$ and $\left.L_{2}\right)$. Task $T_{3}$ is bound to Node 1 , while tasks $T_{1}$ and $T_{4}$ are bound to Node 2 (we have no task $T_{2}$ in this example, we introduce a task $T_{2}$ later in the example in Section 3). $P_{i}$ is the priority of task $T_{i}$. In our notation, the higher the Task's subscript, the higher its priority so $P_{1}<P_{3}<P_{4}$.

In the example, tasks $T_{1}, T_{3}$ and $T_{4}$ execute the following sequence of steps.

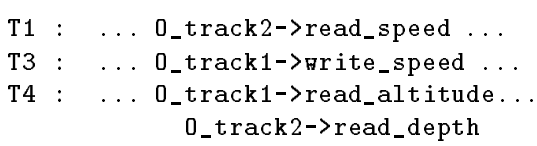

Object $O_{\text {track } 1}$ and its lock $L_{1}$ are bound to Node 1. Object $O_{\text {track } 2}$ and its lock $L_{2}$ are bound to Node 2. The priority ceilings of each lock, and the normal execution priority of each critical section thread are listed in the tables of Figure 1.

The following execution sequences demonstrate several aspects of the DPCP including priority inheritance and several forms of blocking of higher priority tasks by lower priority tasks.

- At time t0, task $T_{1}$ arrives on Node 2 and begins execution. Similarly, task $T_{3}$ begins execution on Node 1.

\begin{tabular}{|c|c|c|}
\hline \multicolumn{3}{|c|}{ Priority Ceilings of Locks } \\
\hline & Lock & $\mathbf{P C}$ \\
\hline & (Global) & $4+4=8$ \\
\hline & 2 (Local) & 4 \\
\hline Norr & ll Executi & $\overline{\text { Priorities of }}$ \\
\hline Task & CS Lock & Priority \\
\hline$\overline{T_{1}}$ & $L_{1}$ & 1 \\
\hline$\overline{T_{3}}$ & $L_{2}$ & $3+4=7$ \\
\hline$T_{4}$ & $\begin{array}{l}L_{1} \\
L_{2}\end{array}$ & $\begin{array}{c}4+4=8 \\
4\end{array}$ \\
\hline
\end{tabular}

Figure 1: Priority Ceilings and Execution Priorities In DPCP Example

- At time t1, task $T_{1}$ gets local lock $L_{2}$ on Node 2 and begins execution of LCS at its normal execution priority of $P_{1}$. Task $T_{3}$ gets the global lock $L_{1}$ on Node 1 and begins execution of its GCS at its normal execution priority of $P_{3}+P G$.

- At time t2, task $T_{4}$ arrives on Node 2 and preempts $T_{1}$. Task $T_{3}$ continues its execution of its GCS on Node 1.

- At time t3, task $T_{4}$ requests global lock $L_{1}$. Since the priority of $T_{4}$ 's GCS $(4+4=8)$ is not greater than the priority ceiling of the held lock $L_{1}(8)$, $T_{4}$ is blocked and $T_{3}$ continues its GCS execution at the inherited priority of $4+4=8$. Task $T_{1}$ resumes its execution of its LCS at Node 2.

- At time t4, task $T_{3}$ completes the execution of its GCS, releases global lock $L_{1}$, and resumes its own priority. Task $T_{4}$ gets global lock $L_{1}$ on Node 1 and begins execution of its GCS at its normal execution priority of $4+4=8$. Task $T_{3}$ is preempted by the higher priority $T_{4}$ 's GCS. Task $T_{1}$ continues the execution of its LCS at Node 2.

- At time t5, task $T_{4}$ completes the execution of its GCS and releases global lock $L_{1}$. Task $T_{3}$ resumes its execution on Node 1. $T_{4}$ attempts to get lock $L_{2}$. However, the priority of $T_{4}(4)$ is not greater than the priority ceiling of the held lock $L_{2}$ (4), so $T_{4}$ is blocked and $T_{1}$ continues its execution with inherited priority of 4 .

- At time t6, task $T_{1}$ completes the execution of its LCS and releases the lock $L_{2}$ and resumes its own assigned priority of 1 . Task $T_{4}$ gets the local lock $L_{2}$ on Node 2 and begins its execution.

- On completion of execution of task $T_{4}$ at t9, task $T_{1}$ resumes its execution; it and $T_{3}$ complete later. 
Note the blocking and priority inheritance that occurred at times $\mathrm{t} 3$ and $t 5$. Although the DPCP introduces new sources of blocking [4], for each source that was not present in the PCP protocols, Rajkumar has shown that the blocking is finite and that DPCP prevents deadlock [4].

Summary of Previous PCPs. In this section we have summarized how the basic PCP and the DPCP work by placing a single ceiling on an entire object, thereby placing an exclusive lock on that object. The Read/Write PCP places two ceilings on an object, thus allowing many readers to an object at any given time and limiting access to only one writer. In the next section we describe how we have introduced affected set semantics to improve concurrency in a single-node object-oriented system by placing multiple priority ceilings on each object - one for each method. Section 4 then describes how we do the same in a distributed system.

\section{Affected Set Priority Ceiling Proto- col.}

This section describes the Affected Set Priority Ceiling (ASPC) protocol, which uses the affected sets [1] of each method of an object to determine the compatibilities of the methods of the object, which in turn establishes priority ceilings for each method.

Using affected set semantics, the critical section requires a method lock. Thus, the ASPC protocol assigns a conflict priority ceiling to each method of each object:

The conflict priority ceiling of a method $m$ is the priority of the highest priority transaction that will ever lock a method that is not compatible with method $m$; where compatibility is defined by affected set semantics.

In order to determine the priority ceilings used in the ASPC protocol, the following four sub-steps to Step 1 in Section 2.2 must be performed:

1a Determine the read/write affected sets for each method.

1b Determine the compatibilities of the methods using the affected sets.

1c Determine the highest priority transaction that will access each method. 1d Determine the conflict priority ceiling for each method using the information from Steps 2 and 3.

At run-time, the priority ceilings are used the same way as in the Original PC and the Read/Write PC protocols: The ASPCP allows a transaction T to receive a lock on a method if and only if the priority of transaction $\mathrm{T}$ is strictly higher than the conflict priority ceiling of locks held by all other transactions.

\subsection{ASPCP Example}

Consider how the ASPCP works in the following example of a tracking real-time object-oriented database with two data objects $O_{\text {track } 1}$ and $O_{\text {track } 2}$ :

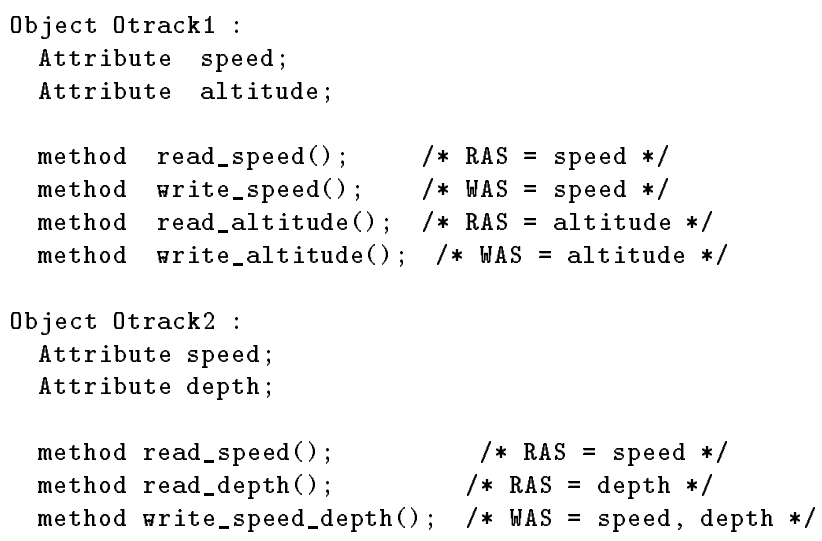

PC Step 1a establishes the read affected sets (RAS) and write affected sets (WAS) of each method, which are also shown with the objects above. For simplicity, these objects were defined to have distinct read and write methods. However, methods are not restricted to this behavior. They can be any user-defined method on the object. Notice that object $O_{\text {track } 1}$ has separate methods to write each attribute, while $O_{\text {track } 2}$ has a method that writes to two attributes.

PC Step 1b establishes the method compatibilities using affected set semantics. These method compatibilities are expressed in the table of YES and NO values of Figure 2. Notice in the table that using affected set semantics, two methods may interact concurrently if they are only reading attributes, or if they are accessing different attributes. Also notice that methods that write to the same attributes may not execute concurrently.

To establish the conflict priority ceilings, the transactions must be examined. Consider four transactions, $T_{1}, T_{2}, T_{3}$, and $T_{4}$, where the transaction's subscript indicates its priority $(1=$ lowest, $4=$ highest $)$. The transactions share objects $O_{\text {track } 1}$ and $O_{\text {track } 2}$ as follows: 


\begin{tabular}{||r|c|c|c|c||}
\hline \hline \multicolumn{5}{||c||}{ Object $O_{\text {track } 1}$} \\
\hline method & read_speed & write_speed & read_altitude & write_altitude \\
\hline read_speed & YES & NO & YES & YES \\
\hline write_speed & NO & NO & YES & YES \\
\hline read_altitude & YES & YES & YES & NO \\
\hline write_altitude & YES & YES & NO & NO \\
\hline \hline
\end{tabular}

\begin{tabular}{||l|c|c|c||}
\hline \hline \multicolumn{4}{|c||}{ Object $O_{\text {track2 }}$} \\
\hline method & read_speed & read_depth & write_speed_depth \\
\hline read_speed & YES & YES & NO \\
\hline read_depth & YES & YES & NO \\
\hline write_speed_depth & NO & NO & NO \\
\hline \hline
\end{tabular}

Figure 2: Affected Set Compatibilities in Example Objects

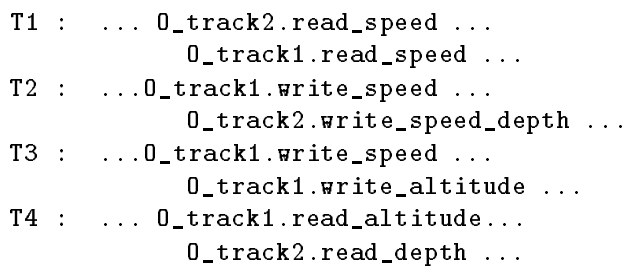

PC Step 1c establishes the highest priority transaction that will invoke each method. PC Step 1d uses this information to determine the conflict priority ceiling for each method. Figure 3 shows the results of PC Steps $1 \mathrm{c}$ and $1 \mathrm{~d}$ for our example. For comparison, it also displays the priority ceilings that would be used by the previous priority ceiling protocols. The determination of the conflict priority ceiling of object $O_{\text {track1's method read_altitude requires identifying all }}$ methods in the compatibility table that conflict with it. From Figure 2 we see that only the write_altitude method conflicts with read_altitude. The conflict priority ceiling of read_altitude is therefore set to the priority of the highest priority transaction that will use write_altitude, which is 3 . The other conflict priority ceilings are set in a similar way.

Figure 4 shows one possible concurrent execution of the transactions using each of the three PC protocols. In all three executions, at time t0, $T_{1}$ starts executing, and at time $\mathrm{t} 1$, is granted a lock, since no other transactions currently hold locks. $T_{2}$ enters the system at time $\mathrm{t} 2$ and preempts $T_{1}$ from the CPU. At time t3, $T_{2}$ attempts to acquire a lock. In all three cases, $T_{2}$ is denied the request since its priority is not greater than the priority ceiling of the lock held by $T_{1}$. Note that this prevents a possible deadlock from occurring between $T_{1}$ and $T_{2}$. At this point, the three protocols begin to differ in their executions, due to the different ceilings that they use. The Original PC protocol (part A) continues to prevent higher priority transactions from acquiring locks until $T_{1}$ releases its locks at time 8. The Read/Write PC protocol (part B) and the ASPCP (part C) allow $T_{3}$ to acquire its lock at time 5 , because $T_{3}$ 's priority is greater than the ceiling of lock held by $T_{1}$ (that lock's priority ceiling is 2 ). When the highest priority transaction, $T_{4}$, enters the system and tries to acquire a lock at time 7 , it is blocked in the Original PC protocol and in the Read/Write PC protocol. On the other hand, the ASPCP allows $T_{4}$ to acquire the lock.

\subsection{ASPCP Properties}

As with the previous priority ceiling protocols, the ASPC protocol bounds priority inversion, prevents deadlock, and produces serializable schedules of object operations. Here we present informal proofs of these claims, which are based on the analogous proofs of the original priority ceiling protocol. Notice that the proofs do not rely on how the priority ceilings are determined, and so the more formal proofs found in [4] apply to the ASPC protocol as well.

\section{Deadlock Prevention.}

Theorem 3.1 The ASPC protocol prevents deadlock.

Proof: Informally, our proof of deadlock prevention is based on the fact that the proofs of deadlock prevention for PC protocols in [4] do not rely on how the priority ceilings are determined. Therefore, with minor adjustments for terminology, a similar proof to those given in [4] proves that the ASPC protocol prevents deadlock. Basically, since the ASPC protocol orders the locks, and maintains this order using PC Step 2, a circular wait cannot occur. Since a circular wait is one of the necessary conditions for deadlock in lock-based systems such as we have described, deadlock cannot occur using the ASPC protocol. The complete proof for deadlock prevention in the ASPC protocol is given in [5].

Bounded Priority Inversion.

Theorem 3.2 Under the ASPC protocol, a transaction $T$ can be blocked by at most a critical section of one 


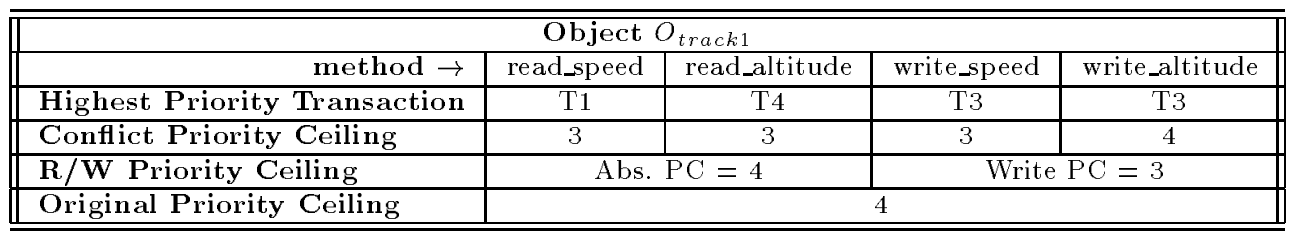

\begin{tabular}{||l|c|c|c||}
\hline \hline \multicolumn{4}{|c||}{ Ob.ject $O_{t r a c k 2}$} \\
\hline \multicolumn{1}{||c|}{ method $\rightarrow$} & read_speed & read_depth & write_speed_depth \\
\hline Highest Priority Transaction & $\mathrm{T} 1$ & $\mathrm{~T} 4$ & $\mathrm{~T} 2$ \\
\hline Conflict Priority Ceiling & 2 & 2 & 4 \\
\hline R/W Priority Ceiling & \multicolumn{2}{c||}{ Abs. PC $=4$} & Write PC $=2$ \\
\hline Original Priority Ceiling & \multicolumn{4}{c||}{4} \\
\hline \hline
\end{tabular}

Figure 3: Priority Ceilings in Tracking Example

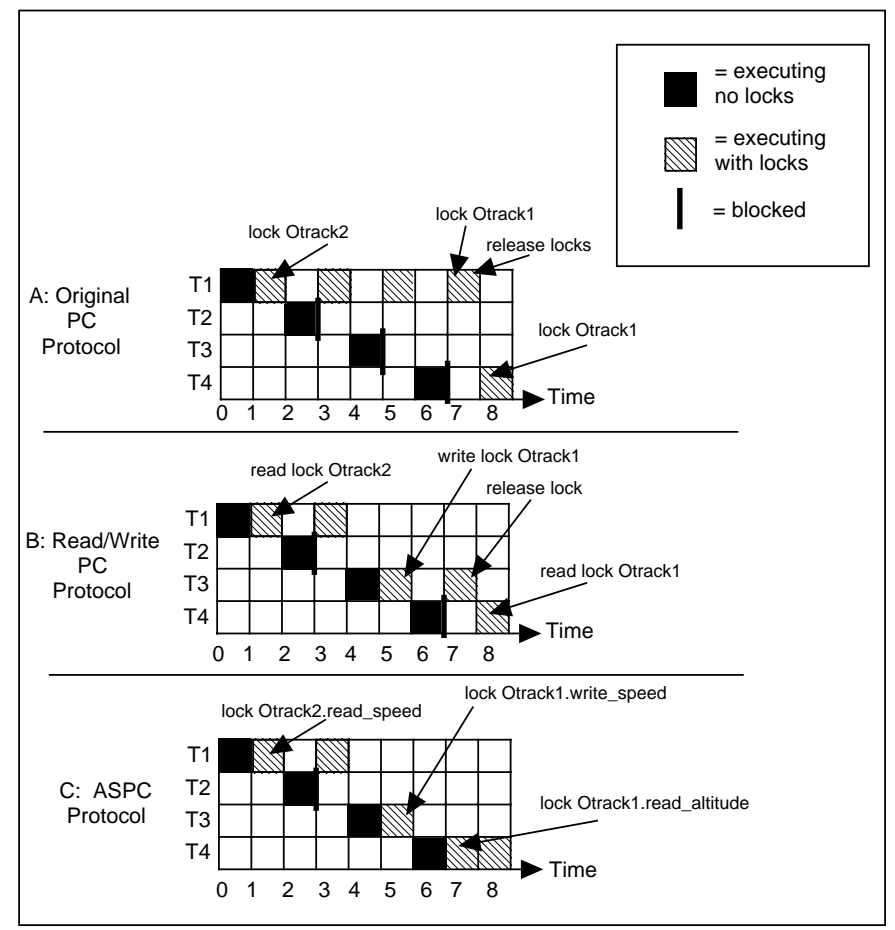

Figure 4: Executions of the Example Transactions Under the Three Priority Ceiling Protocols 
lower priority transaction.

Proof: The proofs of bounded priority inversion in the other PC protocols given in [4] rely on PC Step 2 and PC Step 3 (see Section 2.2), which are common to all priority ceiling protocols, including the ASPC protocol. Again, since the proofs given in [4] do not rely on how the priority ceilings are determined, this proof is similar to those proofs. The complete proof for priority inversion bound using the ASPC protocol is given in $[5]$.

Increased Potential Concurrency. The example of Figure 4 shows how the ASPCP lowers ceilings, which reduces blocking and increases concurrency.

Theorem 3.3 The ASPCP never decreases concurrency compared to the basic PCP technique.

Proof: The priority ceiling used by the PCP is the maximum of the conflict priority ceilings of the ASPCP. Since lower priority ceilings can only mean less blocking time, concurrency can only increase when ASPCP is used instead of PCP.

Serializable Execution.

Theorem 3.4 The ASPCP enforces serializable schedules of method operations for each object.

Proof: Under any PCP, a concurrent access is allowed only if the requesting transaction has a priority higher than the priority ceiling of all held locks. In the ASPCP the priority ceiling of a lock is determined by the priority of transactions accessing conflicting locks. Thus, a lock will not be granted if a conflicting lock is currently held. Badrinath and Ramamritham showed that by defining conflict using affected set semantics, an object is ensured a serializable schedule of method operations [1]. Thus, since ASPCP defines conflict with affected set semantics and denies conflicting locks, it produces a serialable schedule of method operations on each object.

Note that the above theorem discusses serializability of method operations within an object, and not the more global notion of transaction serializability. Twophase locking of method locks can be used to ensure transaction serializability.

\section{Distributed Affected Set Priority Ceiling Protocol}

For concurrency control in distributed real-time object-oriented systems, we have developed the DASPCP that combines techniques from Rajkumar's DPCP, and our ASPCP. In particular, just as the ASPCP uses the PCP mechanism but with the lock granularity at the object method level, the DASPCP uses the DPCP mechanism with its lock granularity at the object method level.
The DASPCP uses the same definition of priority ceilings as does the ASPCP: The priority ceiling of a method $m$ of an object is the highest priority of a transaction that will ever lock a method that is not compatible with method $m$; where compatibility is defined by affected set semantics. The DASPCP also uses the DPCP priority assignment so that GCS's execute at the priority of the requesting task plus the base priority ceiling, PG, of the system (see Section 2.3). Note that these priority ceilings cause the reduced blocking found in the DASPCP compared to the DPCP. The DASPCP also follows the basic steps of all priority ceiling protocols (see Section 2.2).

\subsection{DASPCP Example}

To illustrate the DASPCP, consider the example that was introduced in Section 2.3 then augmented in Section 3.1. The DASPCP priority ceilings are shown in Figure 5. In this figure, the priority ceilings of ob-

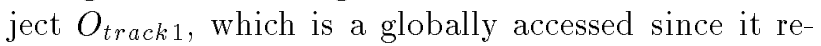
sides on Node 1 and is accessed by $T_{1}$ and $T_{4}$ on Node 2 , are shown as the sum of the highest priority of a task that accesses a conflicting method plus the base priority ceiling, $\mathrm{PG}$ (we have chosen $\mathrm{PG}=4$, the highest priority of any task in the system).

Consider the following execution sequence.

- At time t0, task $T_{1}$ arrives on Node 2 and begins its execution. Similarly, task $T_{3}$ begins execution on Node 1.

- At time t1, task $T_{1}$ gets local lock on $O_{\text {track } 2} \rightarrow$ read_speed on Node 2 and begins execution of its LCS at its normal execution priority of 1 . Task $T_{3}$ gets the global lock on $O_{\text {track } 1} \rightarrow$ write_speed on Node 1 and begins execution of its GCS at its normal execution priority of $3+4=7$.

- At time t2, task $T_{4}$ arrives on Node 2 and preempts $T_{1}$. Task $T_{3}$ continues execution of its GCS.

- At time t3, task $T_{4}$ requests the global lock on $O_{\text {track } 1} \rightarrow$ read_altitude. Since $T_{4}$ 's GCS priority $(4+4=8)$, is higher than the priority ceiling of $O_{\text {track } 1} \rightarrow$ write_speed $(3+4=7)$, it gets the lock on $O_{\text {track } 1} \rightarrow$ read_altitude and preempts $T_{3}$ 's GCS. Task $T_{1}$ continues the execution of its LCS at Node 2.

- At time t4, task $T_{4}$ completes the execution of its GCS and releases the global lock on $O_{\text {track } 1} \rightarrow$ read_altitude. Task $T_{3}$ resumes the execution of its GCS with $O_{\text {track } 1} \rightarrow$ write_speed. Task $T_{4}$ requests a local lock on $O_{\text {track } 2} \rightarrow$ read_depth. 


\begin{tabular}{||l|c|c|c|c||}
\hline \hline \multicolumn{5}{|c||}{ Object $O_{\text {track } 1}$} \\
\hline \multicolumn{1}{||c|}{ method $\rightarrow$} & read_speed & read_altitude & write_speed & write_altitude \\
\hline Highest Priority Transaction & T1 & T4 & T3 & T3 \\
\hline DASPCP Priority Ceiling & $3+4=7$ & 3 & $3+4=7$ & $4+4=8$ \\
\hline DPCP Priority Ceiling & \multicolumn{5}{c||}{$4+4=8$} \\
\hline \hline
\end{tabular}

\begin{tabular}{||l|c|c|c||}
\hline \hline \multicolumn{4}{|c||}{ Object $O_{t r a c k 2}$} \\
\hline method $\rightarrow$ & read_speed & read_depth & write_speed_depth \\
\hline Highest Priority Transaction & T1 & T4 & T2 \\
\hline DASPCP Priority Ceiling & 2 & 2 & 4 \\
\hline DPCP Priority Ceiling & \multicolumn{4}{c||}{4} \\
\hline \hline
\end{tabular}

\begin{tabular}{||c|c|c||}
\hline \hline \multicolumn{3}{||c||}{ Normal Execution Priorities of Methods } \\
\hline Task & Method & Priority \\
\hline$T_{1}$ & Otrack $1 \rightarrow$ read_speed & $1+4=5$ \\
& Otrack $2 \rightarrow$ read_speed & 1 \\
\hline$T_{3}$ & Otrack $1 \rightarrow$ write_speed & $3+4=7$ \\
& Otrack $1 \rightarrow$ write_altitude & 3 \\
\hline$T_{4}$ & Otrack $1 \rightarrow$ read_altitude & $4+4=8$ \\
& Otrack $2 \rightarrow$ read_depth & 4 \\
\hline \hline
\end{tabular}

Figure 5: Priority Ceilings and Execution Priorities in Distributed Tracking Example

Since $T_{4}$ 's priority (priority $=4$ ) is higher than the priority ceiling of $O_{\text {track } 2} \rightarrow$ read_speed (PC $=2), T_{4}$ gets lock on $O_{\text {track } 2} \rightarrow$ read_depth and preempts task $T_{1}$.

- At time t5, task $T_{3}$ completes the execution of its GCS. Execution on Node 2 remains unchanged.

- At time t7, task $T_{4}$ completes its execution including execution of its LCS with $\mathrm{O}_{\text {track } 2} \rightarrow$ read_depth and releases that lock. Task $T_{1}$ resumes execution of its LCS on $O_{\text {track } 2} \rightarrow$ read_speed on Node 2. Tasks $T_{1}$ and $T_{3}$ complete their executions at some later times.

Notice that two blockings of high priority task $T_{4}$ that occurred in the DPCP example of Section 2.3 (the blocking on the global lock at time $t 3$ and the blocking on the local lock at time t5) are alleviated under the DASPCP.

\subsection{DASPCP Properties}

As we did with the ASPCP, we present informal proofs that the DASPCP bounds priority inversion, prevents deadlock, and never decreases concurrency compared to the DPCP. Again, the first two proofs follow Rajkumar's proofs in [4].

\section{Deadlock Prevention.}

Theorem 4.1 The DASPC protocol prevents deadlock. Proof: Informally, our proof of deadlock prevention is based on Rajkumar's results [4]. Since a job can not deadlock with itself, it can deadlock with other jobs.
Since the nesting of GCSs and LCSs is prohibited, access to gcs's and lcs's cannot occur within the same critical section. Since each global and local semaphore is accessed only by a single processor, deadlocks can't occur across processor boundaries. The only possibility, we have not considered yet, is a deadlock within a processor. We showed in Section 3.2 that the ASPCP used on each processor excludes deadlock on that node.

\section{Bounded Priority Inversion.}

Theorem 4.2Under the DASPC protocol, the blocking experienced by a task $T$ is finite.

Proof: We partition blocking into three types and show that each type is finite.

1. A Task's Execution on its Local Node. Task T can be blocked for the duration of at most $\left(n^{G}+1\right)$ local critical sections of lower priority jobs bound to the same processor as $T$. Here $n^{G}$ is the number of GCS's executed by $T$ at remote processors during its period. To see this, realize that task $T$ suspends itself $n^{G}$ times during one period as its execution is transferred to the GCS at the remote node. Task $T$ may be blocked every time it attempts to resume its execution after returning from the GCS. Under the ASPCP, the blocking time of each resumption is limited by a longest critical section of one low priority task. Thus, T's execution on its local node has bounded priority inversion.

2. Task T's GCSs. For every outermost GCS that 
task $T$ enters at a remote processor, $T$ can be blocked for the duration of one longest GCS of a lower priority priority job executing its GCS at the same node. This follows from the fact that under ASPCP on that node, the GCSs as tasks on that node are limited to blocking by at most one lower priority GCS. Thus, each GCS of $T$ has bounded blocking time.

3. Blocking by Remote Tasks. Task $T$ can be preempted by any task $T_{i}$ residing at a remote node and accessing GCSs on T's host node, as well as by higher priority tasks executing their GCS at the same remote node used by T's GCSs. The execution times of GCSs are finite; the number of tasks is finite; the periods are finite; therefore there may not be an infinite repetition of a task $T_{i}$ during one period of $T$. Thus, the blocking due to remote tasks is finite.

Since all types of blocking are finite, the overall blocking for task $T$ is finite.

Increased Potential Concurrency. The example of Section 4.1 shows how the DASPCP lowers ceilings, which reduces blocking, thereby increasing concurrency.

Theorem 4.3 The DASPCP never decreases concurrency compared to the basic DPCP technique.

Proof: The priority ceiling used by the DPCP is the maximum of the conflict priority ceilings of the DASPCP. Since lower priority ceilings can only mean less blocking time, concurrency can only increase when DASPCP is used instead of DPCP.

\section{Serializable Execution.}

Theorem 4.4 The DASPCP enforces a serializable schedule of method operations for each object.

Proof: The proof is similar to that of Theorem 3.4 since the DASPCP also defines priority ceilings based on conflict, and conflict is still defined by affected set semantics.

\section{Conclusion}

This paper has presented the Affected Set Priority Ceiling Protocols (ASPCP) for concurrency control in real-time object-oriented systems. It showed that these protocols: enforce logical consistency by serializable access to objects; prevent deadlock; bound priority inversion; and provide more potential concurrency in object-oriented systems compared to other priority ceiling techniques.

The ASPCP is suitable for single node systems such as real-time object-oriented databases. We have built the ASPCP into a shared main memory realtime object-oriented database [5]. The DASPCP is suitable for controlling access to distributed objects. We have implemented a Basic Priority Ceiling Protocol within the CORBA Concurrency Control Service interface as part of a Real-Time CORBA prototype extension to the commercial Orbix CORBA system from Iona Technologies [6]. We are currently implementing the DASPCP in a static Real-Time CORBA ORB with a global Deadline-Monotonic priority assignment to allow for global analysis of real-time requirements across CORBA. In both cases, the mechanisms to enforce the ASPCP protocols were straightforward and efficient to implement.

The drawbacks of the ASPCP protocols, like all PCP protocols, center on the strong requirement that all task/transactions and their behavior be known $a$ priori. For some real-time applications, this assumption is reasonable, for others it is not. Also, PCP protocols potentially allow less concurrency than straight read/write locking and many other traditional concurrency control techniques. However, the PCP protocols introduce the reduced concurrency due to extra denials of locks that are the price for the deadlock prevention and priority inversion bounds that they provide.

In real-time object-oriented systems where tasks are known a priori, a simple and efficient implementation of either ASPCP or DASPCP will yield logical consistency of object while supporting real-time analysis through deadlock prevention and priority inversion bounding.

\section{References}

[1] B. Badrinath and K. Ramamritham. Synchronizing transactions on objects. IEEE Transactions on Computers, 37(5):541-547, May 1988.

[2] L. C. DiPippo and V. F. Wolfe. Object-based semantic real-time concurrency control with bounded imprecision. IEEE Transactions on Knowledge and Data Engineering, 9(1):135-147, Jan-Feb 1997.

[3] The Real-time Platform Special Interest Group of the OMG. CORBA/RT white paper. ftp site: ftp://ftp.osaf.org/whitepaper/Tempa4.doc, Dec 1996.

[4] R. Rajkumar. Synchronization in Real-Time Systems: A Priority Inheritance Approach. Kluwer Academic Publishers, Boston, MA, 1991.

[5] M. Squadrito. Extending the priority ceiling protocol using read/write affected set semantics. Master's thesis, Dept. of Computer Science, The University of Rhode Island, April 1996.

[6] V. F. Wolfe, L. C. DiPippo, R. Ginis, M. Squadrito, S. Wohlever, I. Zykh, and R. Johnston. Real-time CORBA. In Proceedings of the Third IEEE Real-Time Technology and Applications Symposium, June 1997. 